\title{
Randomised Controlled Trial for the Efficacy of Cervical Lateral Glide Mobilisation in the Management of Cervicobrachial Pain
}

\author{
Emma Salt ${ }^{*}$, Sue Kelly², Andrew Soundy ${ }^{2}$ \\ ${ }^{1}$ Physiotherapy Department, Queen's Hospital Foundation Trust, Burton on Trent, UK \\ ${ }^{2}$ School of Sport, Exercise and Rehabilitation Sciences, The University of Birmingham, Birmingham, UK \\ Email: *emma.salt@nhs.net
}

Received 26 May 2016; accepted 31 July 2016; published 3 August 2016

Copyright (C) 2016 by authors and Scientific Research Publishing Inc.

This work is licensed under the Creative Commons Attribution International License (CC BY). http://creativecommons.org/licenses/by/4.0/

(c) (i) Open Access

\begin{abstract}
Objectives: To investigate the long-term efficacy of lateral glide mobilisation for patients with chronic Cervicobrachial Pain (CP). Methods: A randomised controlled trial which involved ninetynine participants with chronic CP. Participants were randomised to receive either the lateral glide with self-management $(n=49)$ or self-management alone $(n=50)$. Four assessments were made (at baseline and 6, 26 and 52 weeks post intervention). The primary outcome measure was the Visual Analogue Scale (VAS) for pain. Patient perceived recovery used the Global Rating of Change score (GROC). Functional outcomes included the Neck and Upper Limb Index score (NULI) and the Short-From 36 (SF36). Costs and reported number of harmful effects in response to intervention were evaluated. An intention to treat approach was followed for data analysis. Results: No statistically significant between-group differences were found for pain (using VAS) in the short-term at six weeks $(p=0.52 ; 95 \% \mathrm{CI}-14.72$ to 7.44$)$ or long-term at one year $(p=0.37 ; 95 \% \mathrm{CI}-17.76$ to 6.61) post-intervention. The VAS outcomes correlated well with GROC scores $(p<0.001)$. There was a statistically significant difference in NULI scores favouring self-management alone $(p=$ $0.03)$, but no between-group differences for SF36 $(p=0.07)$. The cost of providing lateral glide and self-management was twice that of providing self-management alone. Minor harm was reported in both groups, with $11 \%$ more harm being associated with the lateral glide. Conclusion: In patients with chronic CP, the addition of a lateral-glide mobilization to a self-management program did not produce improved outcomes and resulted in higher health-care costs.
\end{abstract}

\section{Keywords}

Cervical Radiculopathy, Physiotherapy, Manual Therapy

\footnotetext{
${ }^{*}$ Corresponding author.
}

How to cite this paper: Salt, E., Kelly, S. and Soundy, A. (2016) Randomised Controlled Trial for the Efficacy of Cervical Lateral Glide Mobilisation in the Management of Cervicobrachial Pain. Open Journal of Therapy and Rehabilitation, 4, $132-145$. http://dx.doi.org/10.4236/ojtr.2016.43012 


\section{Introduction}

Cervicobrachial Pain (CP) is defined as the presence of upper-quadrant pain associated with cervical spine pain [1]. CP can therefore be referred to the arm from somatic structures or radiate to the upper limb through neuropathic mechanisms. Numerous classifications have been reported, including cervicobrachial pain syndrome, cervical radiculopathy and neck and arm pain. For this study, CP is defined as "the presence of arm pain associated with cervical spine pain" which might include both somatic referred and/or radiating neuropathic mechanisms. $\mathrm{CP}$ has been estimated to affect approximately $30 \%$ of individuals at some time in their lives and features in $60 \%$ of chronic whiplash presentations [2]. It is reported to be twice as common as neck pain in isolation [3] and frequently accompanies cervicogenic headache [4].

Conservative management has been advocated as the initial treatment of choice for the majority of patients with CP [3] [5]. A systematic review on conservative treatments for CP [1] identified that there were potential benefits indicated in the provision of manual therapy and exercise to reduce pain, although, a statistically significant effect was not identified within the meta-analyses. The inclusion of a wide range of manual therapy techniques was attributed as one factor that could have a major influence on the significance of these findings. Indeed, whilst some techniques might provide enhanced therapeutic effect, others may have little therapeutic benefit. Thus, research is required to evaluate and distinguish the effectiveness of particular manual therapy techniques for specific musculoskeletal conditions, such as CP [5].

The most consistently used manual therapy approach reported in the systematic review was the lateral glide mobilisation technique [1]. The lateral glide as a standalone technique has a good level of evidence that it can have both a positive mechanical and physiological effect on pain immediately post treatment [6]-[10]. Currently, evidence considering the clinical effectiveness of the lateral glide on CP is limited to studies evaluating the short-term efficacy ( $<6$ months) [11] of low methodological quality; [12]. Despite this, clinical texts which inform clinicians are advocating the lateral glide for use in CP [13]. Thus, high quality trials, with longer-term ( $>6$ months) follow up assessments are urgently required [14].

The primary objective (for which the trial was powered) was:

- To establish the long-term efficacy of the lateral glide mobilisation in reducing pain, for patients with chronic CP.

Secondary objectives included:

- To assess the short-term and longitudinal efficacy of the lateral glide mobilisation to reduce pain.

- To assess the short-term efficacy of the lateral glide mobilisation to reduced patient perceived change.

- To assess the longitudinal efficacy of the lateral glide mobilisation to improve function and disability.

- To evaluate the short-term cost-effectiveness of the lateral glide in terms of utilisation of physiotherapy resources.

- To identify short-term risk of harm associated with the lateral glide mobilisation.

\section{Material and Methods}

\subsection{Design}

A prospective, randomised controlled trial was undertaken considering the lateral glide technique for patients with a diagnosis of CP. It was a two-arm design, comparing the efficacy of the lateral glide technique with selfmanagement booklet (Mobilisation group) against a comparator group which only received the self-management booklet (Comparator group). This was a single blind trial with the assessors being masked to intervention.

Treatment allocation was determined using a computer number table generator [15]. An independent researcher generated the random order list. Stratified randomisation procedures were used to balance baseline pain severity scores across the two intervention groups, within each of three classifications of baseline visual analogue pain scores: low 0 - 25, moderate 26 - 74 and high 75 - 100. This approach ensured similarity in pain scores between intervention groups at baseline. The identified treatment allocation was concealed in numbered, opaque envelops. Due to the nature of the intervention only the outcome assessors were blind to intervention received.

The trial obtained ethical approval from The South Staffordshire Local Research Ethics Committee (09/ H1203/45) and was registered with "Current Controlled Trials" (ISRCTN62431186) to enable transparency of the planned methods from the outset [16]. 


\subsection{Participants, Therapists and Centre}

The trial was conducted between July 2009 and August 2011, at one hospital site located in the west midlands at a NHS Foundation Trust. Participants were recruited from outpatient orthopaedic and physiotherapy departments, through direct appointments. Eligible participants were adults aged between 18 to 65 years, with chronic CP (duration for greater than six weeks) who had adequate knowledge of English language to independently complete the questionnaires and provide informed consent to being involved in the trial and attend all review appointments. CP was defined as pain in the upper quadrant associated with cervical spine pain. Diagnosis was based on patients' subjective presentation of the pain pattern rather than any specific diagnostic criteria. It therefore included patients with somatic referred pain and neurogenic radiating symptoms. Exclusion criteria were bilateral symptoms (due to the unilateral nature of the lateral glide mobilisation technique), symptoms indicative of serious pathology (history of cancer, unexplained weight loss, severe unremitting night pain, general malaise and constant unvarying pain), vascular thoracic outlet syndrome, co-existing upper limb pathology (e.g. shoulder impingement), systemic conditions affecting joints or nerves (e.g. rheumatoid arthritis or multiple sclerosis), previous surgically invasive techniques to the neck, receiving or planned to receive alternative interventions for $\mathrm{CP}$, involved in litigation or already involved in other research studies. The rights of the participants were protected.

Musculoskeletal specialist physiotherapists (UK pay structure band 7 or 8) working in either the musculoskeletal physiotherapy department or orthopaedic out-patient clinics, assessed participants for suitability of inclusion and booked the participant in with a Trial Physiotherapist (UK pay structure band 6) for one week later. Intervention commenced at the first visit to the Trial Physiotherapist.

\subsection{Interventions}

All participants received the self-management booklet. The self-management booklet consisted of three sections. The first section was on behavioural change relating to posture, workplace, breaks and coping. This first section adopted a cognitive behavioural approach which is an important recognised approach for managing pain [17][19]. The second section was home exercise focusing on mobilising the neck and shoulder and cervical stabilising exercise for the neck. The final section was advice on staying active, relaxation and self-help strategies. The Trial Physiotherapists were requested to spend a consistent time (approximately 15 minutes) on the booklet with each participant at their first interventional appointment. This was to ensure, as far as possible, that initial provision of self-management was delivered equally across trial groups. The Trial Physiotherapist and participant jointly determined the amount of further interventions (up to six interventions) to clarify information or check exercises (regardless of group allocation) as per standard practice at the site where the trial was held.

In addition to the self-management intervention, the Mobilisation group received lateral glide mobilisations. The lateral glide mobilisation was a manual treatment, involving an oscillatory movement applied to the neck with an element of traction. Consistent findings from studies have supported that the lateral glide can induce a hypoalgesic effect on neck and arm pain [7] [8]. It is thought that the technique stimulates cervical afferents which can affect pain processes at spinal cord and cortical levels [20] [21]. Methods used to deliver the glide in previous research have been inconsistent. There has been variability in the position the patient receives the mobilisation and the method of physical application. In this trial the lateral glide mobilisations were performed with the patient in a supine position, with an oscillatory glide directed away from the side of pain, for duration of 60 seconds and for three repetitions (Figure 1).

A grade III mobilisation (into resistance) was used at the level of the fifth on sixth cervical vertebra, which is consistent with previous research [2] [11] [22]. From past literature there have been no explanations to justify dose of treatment and no clear indications whether fewer or more mobilisations affected outcomes. The mean duration of treatment across studies was 60 seconds and the most frequently used level of mobilisation for the lateral glide was fifth relative to sixth cervical vertebrae [6]-[8] [10] [12] [23] [24]. There is some evidence that the largest amplitude of mid to lower cervical spine motion occurs between the fifth and sixth vertebrae [25] [26], therefore it is possible that mobilisation to this level has the greatest impact on mechanoreceptor stimulation. It has been theorised that an increased afferent input from mechanoreceptor stimulation might result in greater changes to spinal cord hyper excitability leading to an increased stimulation of the periaqueductal gray at the level of the brain. This results in an increased descending cortical control pain inhibition [21].

Current evidence suggests that equivalent effects result from manual therapy delivered to either a symptomatic 


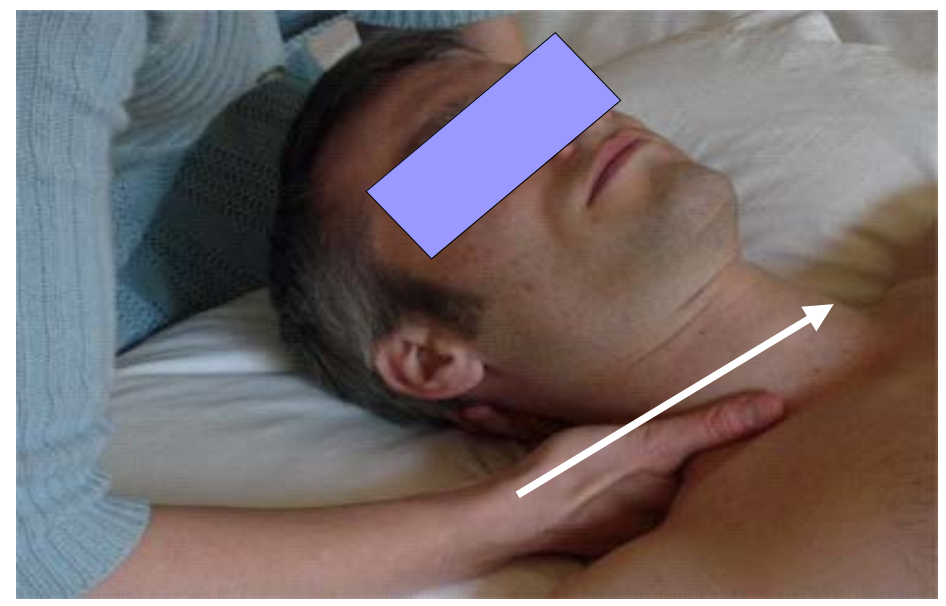

Figure 1. Lateral glide mobilisation technique for right cervicobrachial pain. Footnote: The arrow shows the direction of movement.

or non-symptomatic level [27] [28]. It has been recommended that, in research, techniques should be specific and reproducible to enable findings to inform practice [13]. Following the study by Sterling et al., [8] the technique was delivered using a whole hand contact on the symptomatic side. The placement and pressure of the whole hand contact was to increase stimulation to the mechanoreceptors of the soft tissue structures, thereby creating a larger effect on the pain modulating mechanisms [20] [21] [29].

The interventions were provided up to a maximum of six weeks treatment, regardless of group allocation. The quantity of intervention per individual was determined by both the Trial Physiotherapist providing the intervention and the participant as per standard practice. Assessments were made on four occasions (at baseline prior to intervention and at 6, 26 and 52 weeks post the first intervention session). The intervention period was the time between commencement of intervention and the first follow-up time point (6 weeks). Six weeks was selected to represent the end of the intervention period, based on participants receiving up to six, weekly appointments. Six or fewer appointments had been identified in the literature as the mean number of appointments needed to achieve clinical effectiveness in neck pain [30] [31].

\subsection{Outcome Measures}

The following outcome measures were selected based on previous guidance [16] [32].

\subsubsection{Pain (Primary Outcome Measure)}

This trial used a visual analogue scale (VAS) which represented the worst pain in their neck and arm over the preceding week. The scale comprised of a $100 \mathrm{~mm}$ horizontal line marked "no pain" at 0 and "worst pain imaginable" at 100. A clinically meaningful difference was taken as $20 \mathrm{~mm}$ as identified in previous research [33].

\subsubsection{Patient Perceived Change in Pain}

The Global Rating of Change Score (GROC) provided an overall perception of change in pain, ranging from -6 (a great deal worse from baseline) to +6 (a great deal better from baseline); with 0 indicating no change [34].

\subsubsection{Function and Disability}

Function and disability was assessed using a condition-specific and generic outcome measure. This allowed detailed information regarding limitations to that condition as well as a more generic evaluation of well-being, respectively [35]. The condition-specific measure used was The Neck and Upper Limb Index (NULI) [36]. This measure has been reported to have high levels of reliability and validity for patients with neck and arm pain [36]. NULI comprised twenty questions, divided into four sub-scales: physical activities, work, psychosocial factors, and, sleep. Scores ranged from 0 to 100 , with high scores indicating worse function/disability. The mental component summary (MCS) of the RAND Short-form 36 (SF-36) version 2 was selected as the generic outcome measure [37]. This summary is scaled from 0 to 100, with higher scores representing good health [37] [38]. 


\subsubsection{Cost}

Physiotherapy utilisation cost was expressed as the mean number of physiotherapy appointments needed per group and calculated in terms of mean monetary value of unit costs for therapist time [39].

\subsubsection{Harm}

Levels of harm were categorised based on the Common Terminology Criteria for Adverse Events [40]: Mild, Moderate or Severe. Harm was expressed as the number (and percentage) of participants who had reported harm associated with an intervention within the six week intervention period.

\section{Data Analysis}

The sample size for the trial was determined through statistical methods using the identified primary end point, (VAS) at 52 weeks, together with evidenced values for i) the minimal clinically meaningful difference in a comparable patient population; ii) an estimate of standard deviation in the population of interest (based on previous pilot study data); iii) the effect size calculated from i) and ii) and, iv) adjustment for participant withdrawal. The sample size was based on a statistical significance level of 0.05 , a power of 0.80 , and an effect size of 0.65 on VAS. This resulted in a total of 68 participants needed at completion of the trial, with 34 participants in each group. An 11\% attrition rate was allowed at each three measurement periods meaning an adjusted sample size of 96 participants needed to be recruited at the beginning of the trial, with 48 participants randomised to each group.

Historically, testing for baseline differences have been used to identify if any between-group differences at baseline are likely to influence results at follow-up [41]. It has been well documented that multiple statistical testing on baseline data can lead to multiplicity [42]. The use of statistical approaches such as Analysis of Covariance (ANCOVA) and Multi-Level Modelling (MLM) that incorporate potential baseline confounders in the statistical models are considered a superior approach to account for any baseline between group variability. Therefore between-group differences in baseline data were not analysed in this study.

An intention to treat approach was followed for the analysis of data. The primary analysis used ANCOVA to test between-group differences at 52 week follow-up (primary long-term end point) and at six week follow-up (secondary short-term end point). Covariates used included gender, age, mental health, chronicity and pain at baseline. In addition, all time points (6, 36 and 52 weeks) were used for a longitudinal analysis using MLM. The addition of MLM enabled any missing data to be accommodated.

Association between patient perceived improvement in pain (GROC) and the primary outcome measure VAS (pain) at six weeks was assessed using Spearman's rho.

Methods for the statistical analyses of data on secondary outcome measures used MLMs for parametric data (NULI and SF-36) and Mann-Whitney tests for between-group comparisons for ordinal data (physiotherapy utilisation). Harm was expressed as a number (and percentage) of participants who reported harm with an intervention. All tests were conducted using a significance level of 5\% and results were used with caution since no adjustment was made for multiple testing. All statistical analyses were completed using SPSS (version 19).

\section{Results}

\subsection{Participant Flow}

Assessment Physiotherapists identified a total of 286 patients with CP who were suitable for physiotherapy. Eligible participants $(n=112)$ were invited to attend the first appointment with a Trial Physiotherapist. Thirteen patients decided not to participate in the trial; 99 gave informed consent and were randomised to receive lateral glide mobilisation and self-management $(n=49 ; 50 \%$; Mobilisation group) or self-management alone ( $n=50$; 50\%; Comparator group). The randomisation procedure resulted in a balance of numbers across groups. One participant in the Mobilisation group withdrew after starting treatment due to dissatisfaction with the intervention because he did not believe that he was actually receiving treatment.

Ninety two percent $(n=46)$ of participants in the Comparator group and $86 \%(n=42)$ in the Mobilisation group completed assessments at the primary end point (52 weeks) (Figure 2).

Baseline demographic data indicated that participants in the two groups had similar characteristics.

The range of occupations and life style behaviours were similar across groups. See Table 1 for a complete breakdown. 


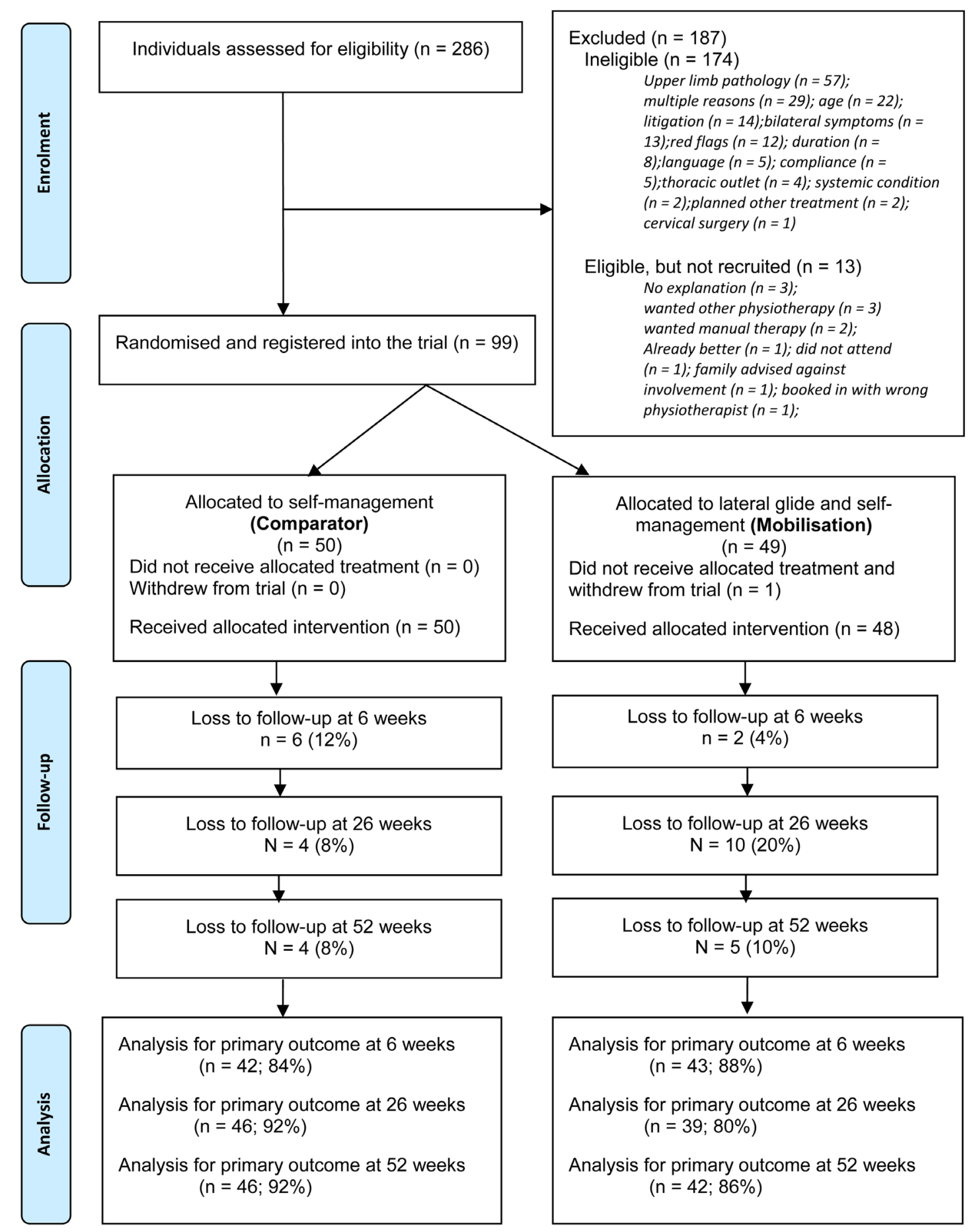

Figure 2. Participant flowchart of the trial (Adapted from Moher et al., 2010).

Baseline clinical characteristics were similar across groups, with no clinically meaningful differences. The initial clinical examination included cervical spine active range of motion. The largest between group differences at baseline was right rotation where mean range was 58 (SD17) degrees for the Mobilization group and 65 (SD20) degrees for the Comparator group. A reduction in right rotation in the Mobilisation group could be due to the fact that slightly more of the Mobilisation group had their right side affected by cervicobrachial pain $(\mathrm{n}=$ 28 ; $57 \%$ ) compared to the comparator group $(n=27 ; 54 \%)$ (Table 2$)$. 
Table 1. Demographic characteristics for randomised participants, at baseline ( $n=99)$.

\begin{tabular}{ccc}
\hline Variable & & Intervention \\
& Comparator $(\mathbf{n}=\mathbf{5 0})$ & Mobilisation (n = 49) \\
\cline { 2 - 3 } Age (years) & Mean (SD) & Mean (SD) \\
& $47(11)$ & $47(11)$ \\
Gender & $\mathrm{n}(\%)$ & $\mathrm{n}(\%)$ \\
Females & & \\
Occupation & $24(47)$ & $27(53)$ \\
Manual worker & & \\
Desk worker & $20(40)$ & $22(45)$ \\
Retired & $14(28)$ & $10(20)$ \\
Unemployed & $3(6)$ & $4(8)$ \\
Other & $3(6)$ & $3(6)$ \\
Sickness (days) & $9(18)$ & $10(20)$ \\
None & & \\
$1-5$ & $35(70)$ & $35(72)$ \\
$6-10$ & $1(2)$ & $0(0)$ \\
$11-15$ & $2(4)$ & $1(2)$ \\
$>16$ & $2(4)$ & $0(0)$ \\
Not applicable & $3(6)$ & $10(20)$ \\
Smoker & $7(14)$ & $3(6)$ \\
Yes & $20(40)$ & $15(30)$ \\
\hline
\end{tabular}

Key: $\mathrm{n}$ = number of participants; $\mathrm{SD}$ = standard deviation.

Table 2. Clinical characteristics at baseline for randomised participants $(n=99)$.

\begin{tabular}{|c|c|c|c|}
\hline & & \multicolumn{2}{|c|}{ Intervention } \\
\hline & & Comparator $(n=50)$ & Mobilisation $(n=49)$ \\
\hline \multicolumn{2}{|l|}{ Variables } & Mean (SD) & Mean (SD) \\
\hline \multicolumn{2}{|l|}{ VAS (pain) } & $65(20)$ & $63(22)$ \\
\hline \multicolumn{2}{|l|}{ SF-36 } & $65(17)$ & $60(18)$ \\
\hline \multicolumn{2}{|l|}{ NULI } & $30(17)$ & $36(19)$ \\
\hline \multicolumn{4}{|c|}{ AROM cervical spine (using inclinometer) } \\
\hline & Flexion & $49(15)$ & $47(13)$ \\
\hline & Extension & $48(14)$ & $46(15)$ \\
\hline & Right rotation & $65(20)$ & $58(17)$ \\
\hline & Left rotation & $64(18)$ & $62(17)$ \\
\hline & & n (\%) & \\
\hline \multicolumn{4}{|c|}{ Chronicity (months) } \\
\hline & $2-3$ & $5(10)$ & $6(12)$ \\
\hline & $>3-6$ & $10(20)$ & $11(22)$ \\
\hline & $>6-12$ & $14(28)$ & $13(25)$ \\
\hline & $>12$ & $21(42)$ & $19(40)$ \\
\hline
\end{tabular}




\begin{tabular}{|c|c|c|c|}
\hline \multicolumn{4}{|l|}{ Continued } \\
\hline ULNE test & Positive & $39(78)$ & $36(73)$ \\
\hline WAD & Yes & $6(12)$ & $5(10)$ \\
\hline Dominant arm & Right & $45(92)$ & $47(96)$ \\
\hline Side Involved & Right & $27(54)$ & $28(57)$ \\
\hline \multicolumn{4}{|l|}{ Distribution } \\
\hline & $\mathrm{C} 4 / 5$ & $22(18)$ & $11(22)$ \\
\hline & $\mathrm{C} 5 / 6$ & $9(44)$ & $29(59)$ \\
\hline & $\mathrm{C} 6 / 7$ & $14(28)$ & $5(10)$ \\
\hline & $\mathrm{C} 7 / \mathrm{T} 1$ & $5(10)$ & $4(8)$ \\
\hline \multicolumn{4}{|l|}{ Dysfunction } \\
\hline & Pain only & $9(18)$ & $11(23)$ \\
\hline & Pain \& sensory change & $27(55)$ & $27(56)$ \\
\hline & Pain, sensory \& motor change & $13(27)$ & $10(8)$ \\
\hline \multicolumn{4}{|l|}{ Preference } \\
\hline & None & $36(72)$ & $36(74)$ \\
\hline & To comparator & $2(4)$ & $2(4)$ \\
\hline & To lateral glide & $12(24)$ & $11(22)$ \\
\hline Previous physiotherapy & Yes & $14(29)$ & 19 (39) \\
\hline Benefit from previous physiotherapy & Yes & $12(92)$ & $9(47)$ \\
\hline
\end{tabular}

Key: AROM = Active Range Of Motion; NULI = Neck and Upper Limb Index; n = number of participants; SF-36 = Short-from 36; SD = standard deviation; ULNE = Upper Limb Nerve Extensibility; VAS = Visual Analogue Scale; WAD = Whiplash associated disorder.

\subsection{Effect of Intervention}

\subsubsection{Pain}

For the primary analysis (long term effect on pain), there was a mean decrease exceeding $20 \mathrm{~mm}$ for both groups for VAS (pain) at 52 week follow-up compared to baseline (Table 3). This indicated that there was a mean clinically meaningful improvement, for participants in both groups. But, there was no statistically significant difference in mean VAS (pain) scores between groups, using a covariate analysis ( $p=0.37$; CI -17.76 to 6.61).

For short-term effect on pain, neither group reached a clinically meaningful reduction in mean pain score (Table 3). There was no statistically significant difference in mean scores on VAS (pain) between groups at follow-up 6 weeks, using a covariate analysis ( $p=0.52$; CI-14.72 to 7.44 ).

Longitudinal analysis of pain after accounting for time and any statistically significant baseline covariates identified within the MLM found no statistically significant difference in mean VAS (pain) scores ( $p=0.867$; CI -5.91 to 7.00 ) between the Comparator and Mobilisation groups (Table 4 and Figure 3).

According to the patient perceived change in pain (GROC) both groups had achieved a clinically meaningful improvement (increase of two points) at 6 week follow-up. There was a moderate association (Spearmans rho $=$ 0.69; $p<0.001$ ) between GROC and VAS (pain) at six weeks (Figure 4). No participant was clinically worse on both outcome measures (VAS (pain) score $>$ or equal to 20 and GROC score < or equal to 2; Figure 4, shaded box B). Twenty-seven of the participants were identified as clinically improved on both outcomes measures (see shaded box A), 13 individuals were in the Mobilisation group.

As the majority $(\mathrm{n}=75 ; 77 \%)$ of participants included in the trial had a positive ULNE (nerve mechano-sensitivity), further statistical tests (using ANCOVA) were conducted on this sub-group. Patients with a positive ULNE had no statistically significant between-group difference for VAS (pain) at six weeks ( $p=0.30 ; 95 \%$ CI -19.02 to 5.94). This indicated that participants with nerve mechano-sensitivity did not have a favourable response to the lateral glide mobilisation. 


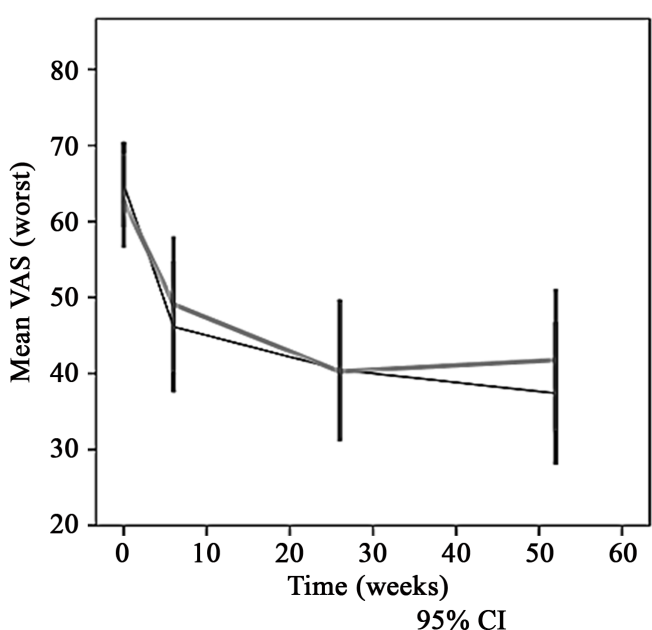

Figure 3. Longitudinal VAS (pain) (MLM). Key: | Comparator group; | Mobilisation group. Footnote: The 95\% CI’s are for each individual intervention and not the difference between interventions.

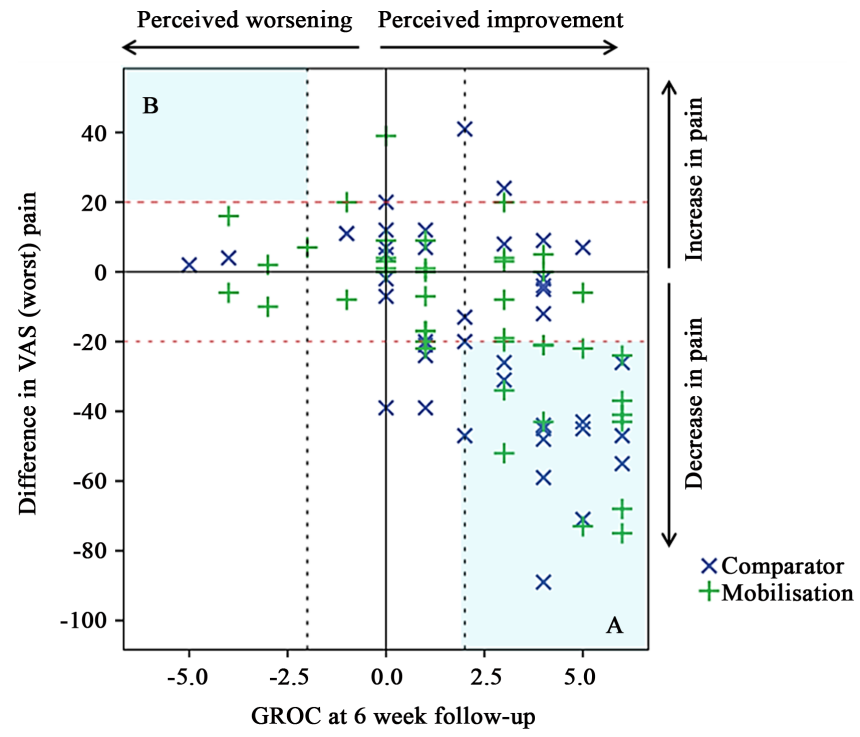

Figure 4. Differences in pain scores for VAS scores (from 6-week follow-up to baseline) compared with GROC at six weeks follow-up. Key: GROC = Global Rating of Change score; VAS = Visual Analogue Scale. Footnote: Horizontal dotted line represents clinically meaningful difference on VAS. Vertical dotted line represents clinically meaningful difference on GROC; Box A indicates a clinically meaningful improvement on both GROC and VAS; Box B indicates a clinically meaningful worsening on both GROC and VAS.

Table 3. Summary statistics for VAS (pain) at each time point.

\begin{tabular}{ccccc}
\hline & \multicolumn{3}{c}{ Intervention } \\
\cline { 2 - 4 } & \multicolumn{2}{c}{ Comparator } & \multicolumn{2}{c}{ Mobilisation } \\
\hline Time point: & $\mathrm{n}(\%)$ & $\mathrm{Mean}(\mathrm{SD})$ & $\mathrm{n}(\%)$ & $\mathrm{Mean}(\mathrm{SD})$ \\
Baseline & $50(100)$ & $65(20)$ & $49(100)$ & $63(22)$ \\
6 week follow-up & $42(84)$ & $46(28)$ & $43(88)$ & $49(29)$ \\
26 week follow-up & $46(92)$ & $40(31)$ & $39(80)$ & $40(28)$ \\
52 week follow-up & $46(92)$ & $37(32)$ & $42(86)$ & $42(30)$ \\
\hline
\end{tabular}

Key: $\mathrm{n}$ = number of participants; $\mathrm{SD}$ = standard deviation. 
Table 4. Results of measures analysed longitudinally (multi-level analyses).

\begin{tabular}{cccccccccccccccc}
\hline & \multicolumn{4}{c}{ Baseline } & \multicolumn{2}{c}{ 6 week f-up } & \multicolumn{2}{c}{ 26 week f-up } & \multicolumn{2}{c}{ 52 week f-up } & \multicolumn{2}{c}{$\begin{array}{c}\text { Differences from MLM tests } \\
\text { (Across time) }\end{array}$} \\
\cline { 2 - 4 } & Comp & Mob & Comp & Mob & Comp & Mob & Comp & Mob & $\begin{array}{c}\text { Estimate } \\
\text { of effect }\end{array}$ & CI & p-value \\
\hline VAS (pain) & $65(20)$ & $63(22)$ & $46(28)$ & $49(29)$ & $40(31)$ & $40(28)$ & $37(32)$ & $42(30)$ & 0.96 & -5.91 to 7.00 & 0.87 \\
NULI & $30(17)$ & $36(19)$ & $24(15)$ & $30(22)$ & $19(17)$ & $26(20)$ & $19(17)$ & $26(20)$ & 6.91 & -13.53 to -0.92 & $0.03^{*}$ \\
SF36 & $65(17)$ & $59(18)$ & $66(18)$ & $62(18)$ & $72(15)$ & $64(20)$ & $70(16)$ & $64(19)$ & 5.85 & -0.37 to 12.07 & 0.07 \\
\hline
\end{tabular}

Key: CI = 95\% confidence interval; Comp = Comparator group; $\mathrm{f}$-up = follow-up; Mob = Mobilisation group; MLM = multilevel modelling; NULI = Neck and Upper Limb Index; SF36 = short-form 36; VAS = visual analogue scale. * Significant at $p<0.05$. Values shown are mean and SD.

\subsubsection{Function and Disability}

Longitudinal analysis of function and disability, after accounting for time and statistically significant baseline covariates in the MLM (gender $p=0.04$; chronicity $p=0.04$ ), found that there was a statistically significant difference in mean NULI scores ( $p=0.03$; CI -13.53 to -0.92$)$ between the Comparator and Mobilisation groups, favouring the effectiveness of the Comparative intervention. For SF36 (MCS), between-group differences did not reach a statistical significance ( $p=0.07$; CI -0.37 to 12.07) (Table 4 ).

\subsubsection{Cost-Effectiveness}

The mean number of attended sessions was more than twice as much for the Mobilisation group than the Comparator. This finding was statistically significant $(p \leq 0.01)$. The average 30 minute "unit cost" for a physiotherapist working in secondary care was $£ 32.00$ (Department of Health, 2013). Based on this figure, the average cost in the intervention period was $£ 64.00$ (approximately \$108 US) for participants in the Comparator group, and $£ 160.00$ (approximately $\$ 270$ US) for participants in the mobilisation group.

\subsubsection{Harm}

Neither intervention led directly to any severe or moderate harm during the course of the intervention period. Minor harm was reported during the intervention period in the trial. Minor harm associated with the exercises was $5 \%$ ( $n=5$ of 99 receiving exercises) compared to minor harm associated with the manual therapy which was $16 \%$ ( $\mathrm{n}=8$ of 49 receiving mobilisations).

\section{Discussion}

The analysis at long-term follow-up (52 weeks post baseline), for which the trial was powered, found no evidence of between-group differences in VAS (pain). Consistent findings for other pain outcomes used in this study (GROC) and across time points supported the likelihood that the lateral glide mobilisation, in this instance, did not provide additional effect on pain reduction. Scores from self-report function and disability measures, NULI and SF36 (MCS), were inconclusive. The NULI had a between-group statistically significant result, favouring the Comparator group. SF36 (MCS) between-group scores also found a trend to support the Comparator group, but this did not reach a statistically significant level. Between-group differences were found on costs relating to physiotherapy utilisation, with the mobilisation group requiring approximately double the amount of intervention than that of the Comparator group. Harm associated with the mobilisation was also approximately double that of the comparative intervention. However it is recognised that outcome measures relating to function, disability, cost and harm were not powered for this trial and therefore need to be interpreted with caution. The overall strength of evidence to support recommendation of the use of the lateral glide for cervicobrachial pain in clinical practice is low.

The findings in this study contrast to previous studies that have found beneficial effects from providing the lateral glide mobilisation for the cervicobrachial pain in the short-term [11] [22] [43]. The disparity between findings of this trial and other similar studies [11] [43] could be attributed to methods in the selection process, including the presence of a positive ULNE. The presence of a positive ULNE as a pre-requisite for inclusion [6] [11] [43] was one of the key differences between this trial and other randomised studies evaluating the lateral glide. For this reason, an exploratory sub-group analysis was conducted on participants with a positive ULNE. 
The majority of participants in the trial had a positive ULNE test ( $\mathrm{n}=75$; 77\%) with an equal balance between the two intervention groups (Comparator $n=39$; Mobilisation $n=36$ ). Results from the sub-group analysis found a non-significant difference on VAS (pain) at six weeks ( $p=0.30$; CI -19.02 to 5.94). Whist it was recognised that this analysis was not powered, it does indicate that the inclusion of a positive ULNE was unlikely to account for the differences seen on pain outcomes.

Only one other study clearly specified both the dose and delivery of the lateral glide [11]. Differences between this trial and that of Nee et al. [11] included the following: Nee et al. [11] used a "pulling” technique (as opposed to "pushing" technique) to provide the translatory oscillation. Mobilisations were administered to multiple levels of the cervical spine (as opposed to the one level used in this trial) for two, 30 second doses (compared to three 60 second doses). Although some evidence supported that similar effects can be gained by using different mobilisation approaches in general [27], [28], other studies that have reported the use of multi-level lateral glide mobilisations in their cervicobrachial pain studies [6] [7], [22] have had consistently positive effects on pain as an outcome. It is therefore possible that the difference in approaches to the lateral glide could have accounted for differences in outcome for pain in this trial compared to the study by Nee et al. [11].

Two randomised studies used the lateral glide with other mobilisation techniques as part of a manual therapy package of care [22], [43]. Coppieters et al. [6] and Nee et al. [11] used the lateral glide as a singular mobilisation technique, as was the situation in this trial. Unlike this trial, both Coppieters et al. [6] and Nee et al. [11] found evidence to support the use of the lateral glide technique to reduce cervicobrachial pain to a greater degree than a comparator in the immediate and short-term. For Nee et al. [11], both intervention groups (comparator and mobilisation) were advised to stay active, however only the mobilisation group received the education and exercises as well as the mobilisation, thus, the improvement found in their study might be attributed to the exercise and education component of the physiotherapy intervention rather than the lateral glide component. Coppieters et al. [6] used an ultrasound comparator and gave the lateral glide as the sole intervention (no advice or exercises added). Limitations with their study included methodological flaws (lack of power, unclear selective reporting) which could have biased results. However, this finding along with others, support the immediate effect of the lateral glide in other neck and upper limb disorders [7] [8] [44] [45].

It is recognised that there were some limitations with this study. Cervicobrachial pain might derive from multiple mechanisms. It is possible that the heterogeneity in this study group was a limitation. At the time of designing the study, there was no method available for effective differentiation of sub-categorisation which led to cervicobrachial pain being considered as a single entity.

This was a single-site trial. As participants were recruited over a long duration, results should be justly representative for all patients at this location. Results of this trial are limited to patients who match the characteristics and demographics of those involved in this trial and, therefore, represent a select sample of patients. Data from participants from primary or tertiary centres and in different geographic locations would have led to a better generalisability; however, as the location used serves a population with mean figures for socio-economic UK demographics, it is possible that the results from this study can be considered a fair representation for a wider UK population.

A large proportion of participants received additional treatment prior to 52 weeks ( $=47 ; 47 \%$ ) which introduced additional sources of variation in the intervention actually received by participants in each group. Although additional treatment between six and 52 weeks was seemingly high, there was no statistically significant inter-group difference (for responders at one year) on the use of additional treatment $(p=0.93)$. All participants within the first six weeks received the protocol intervention as the only treatment received. Thus, the analysis at six weeks effectively became a per-protocol analysis and was more likely to reveal the "true" level of efficacy for the lateral glide. Analysis at this follow-up time found no between-group difference on VAS (pain).

The sample size for this trial was based on a power calculation, using a moderate to large effect size (20mm of change) for the primary outcome measure. Moderate effect sizes have been criticised to lead to a type two error (rejecting the hypothesis when in fact it is true) due to insufficient power to detect a small yet meaningful change. If the trial had been powered to detect a smaller effect size (for example, $10 \mathrm{~mm}$ of change), it is possible that a statistically significant between-group difference might have been detected. However, it is questionable how meaningful an effect of less than $20 \mathrm{~mm}$ of change would be.

\section{Conclusion}

The results of this trial did not find that the lateral glide provided any preferential reduction in pain compared to 
a comparator in the short or long-term. This trial has extended the knowledge base for the efficacy of the lateral glide mobilisation on the management of cervicobrachial pain. This is the only trial that has evaluated the value of the lateral glide as a specific mobilisation technique over a prolonged period of time. In doing so, pragmatic problems were encountered including a large number of participants receiving additional treatment which had the potential to introduce a confounding effect at long-term analysis.

\section{Acknowledgements}

The authors would like to thank the Musculoskeletal Association of Chartered Physiotherapists for their grant to support the trial, to the Research and Development team at Queen's Hospital, Burton on Trent for their support with electronic data entry and to Chris Wright for her support and guidance in the development of this trial.

\section{References}

[1] Salt, E., Wright, C. and Kelly, S. (2011) A Systematic Literature Review on the Effectiveness of Non-Invasive Therapy. Manual Therapy, 16, 53-65. http://dx.doi.org/10.1016/j.math.2010.09.005

[2] Sterling, M., Trevleaven, J. and Jull, G. (2002) Responses to a Clinical Test of Mechanical Provocation of Nerve Tissue in Whiplash Associated Disorders. Manual Therapy, 7, 89-94. http://dx.doi.org/10.1054/math.2002.0443

[3] Daffner, S.D., Hilibrand, A.S., Hanscom, B.S., Brislin, B.T., Vaccaro, A.R. and Albert, T.J. (2003) Impact of Neck and Arm Pain on Overall Health Status. Spine, 28, 2030-2035. http://dx.doi.org/10.1097/01.BRS.0000083325.27357.39

[4] Antonaci, F., Bono, G. and Chimento, P. (2006) Diagnosing Cervicogenic Headache. The Journal of Headache and Pain, 7, 145-148. http://dx.doi.org/10.1007/s10194-006-0277-3

[5] Miller, J., Gross, A., D’Sylvia, J., et al. (2010) Manual Therapy and Exercise for Neck Pain: A Systematic Review. Manual Therapy, 15, 334-354. http://dx.doi.org/10.1016/j.math.2010.02.007

[6] Coppieters, M.W., Stappaerts, K., Wouters, L.L., et al. (2003) The Immediate Effects of a Cervical Lateral Glide Treatment Technique in Patients with Cervicobrachial Pain. Journal of Orthopaedic Sports Physical Therapy, 33, 369378. http://dx.doi.org/10.2519/jospt.2003.33.7.369

[7] McClatchie, L., Laprade, J., Martin, S., Jaglal, S.B., Richardson, D. and Agur, A. (2009) Mobilisations of the Asymptomatic Cervical Spine Can Reduce Signs of Shoulder Dysfunction in Adults. Manual Therapy, 14, 369-374. http://dx.doi.org/10.1016/j.math.2008.05.006

[8] Sterling, M., Pedler, A., Chan, C., Ouglisi, M., Vuvan, V. and Vicenzino, B. (2010) Cervical Lateral Glide Increases Nociceptive Flexion Reflex but Not Pressure or Thermal Pain Thresholds in Chronic Whiplash Associated Disorders: A Pilot Randomised Controlled Trial. Manual Therapy, 15, 149-153. http://dx.doi.org/10.1016/j.math.2009.09.004

[9] Vicenzino, B., Gutschlag, F., Collins, D., et al. (1995) An Investigation of the Effects of Spinal Manual Therapy on Forequarter Pressure and Thermal Pain Thresholds and Sympathetic Nervous System Activity in Asymptomatic Subjects: A Preliminary Report. In Shacklock, M., Ed., Moving in on Pain, Butterworth-Heinemann, Australia, 185-193.

[10] Vicenzino, B., Neal, R., Collins, D., et al. (1999) The Displacement, Velocity and Frequency Profile of the Frontal Plane Motion Produced by the Cervical Lateral Glide Treatment Technique. Clinical Biomechanics, 14, 515-521. http://dx.doi.org/10.1016/S0268-0033(99)00011-X

[11] Nee, R.J., Vincenzino, B. and Jull, G.A. (2012) Neural Tissue Management Provides Immediate Clinically Relevant Benefits without Harmful Effects for Patients with Nerve-Related Neck and Arm Pain: A Randomised Trial. Journal of Physiotherapy, 58, 23-31. http://dx.doi.org/10.1016/S1836-9553(12)70069-3

[12] Cowell, I.M. and Phillips, D.R. (2002) Effectiveness of Manipulative Physiotherapy for the Treatment of a Neurogenic Cervicobrachial Pain Syndrome: A Single Case Study_Experimental Design. Manual Therapy, 7, 31-38. http://dx.doi.org/10.1054/math.2001.0429

[13] Jull, G., Sterling, M., Falla, D., et al. (2008) Whiplash, Headache and Neck Pain. In: Elvey, Ed., Churchill Livingstone, London.

[14] Vernon, H. and Humphreys, B.K. (2007) Manual Therapy for Neck Pain: An Overview of Randomized Clinical Trials and Systematic Reviews. Europea Medicophysica, 43, 91-118.

[15] Kelley, K., Clarke, B., Brown, V. and Sitzia, J. (2003) Good Practice in the Conduct and Reporting of Survey Research. International Journal for Quality in Health Care, 15, 261-266. http://dx.doi.org/10.1093/intqhc/mzg031

[16] Moher, D., Hopewell, S., Schulz, K.F. (2010) CONSORT 2010 Explanation and Elaboration: Updated Guidelines for Reporting Parallel Group Randomized Trials. BMJ, 340, c869.

[17] Bosy, D., Etlin, D., Corey, D. and Lee, J.W. (2010) Interdisciplinary Pain Rehabilitation Programme: Description and Evaluation of Outcomes. Physiotherapy Canada, 62, 316-326. http://dx.doi.org/10.3138/physio.62.4.316 
[18] Hansen, I.R., Søgaard, K., Christensen, R., Thomsen, B., Manniche, C. and Juul-Kristensen, B. (2011) Neck Exercises, Physical and Cognitive Behavioural-Graded Activity as a Treatment for Adult Whiplash Patients with Chronic Neck Pain: Design of a Randomised Controlled Trial. BMC Musculoskeletal Disorders, 12, 274. http://dx.doi.org/10.1186/1471-2474-12-274

[19] Peres, M.F. and Lucchetti, G. (2010) Coping Strategies in Chronic Pain. Current Pain and Headache Reports, 14, 331318. http://dx.doi.org/10.1007/s11916-010-0137-3

[20] Bialosky, J.E., Bishop, M.D., Price, D.D., Robinson, M.E. and George, S.Z. (2009) The Mechanisms of Manual Therapy in the Treatment of Musculoskeletal Pain: A Comprehensive Model. Manual Therapy, 14, 531-538. http://dx.doi.org/10.1016/j.math.2008.09.001

[21] Schmid, A., Brunner, F., Wright, A. and Bachmann, L.M. (2008) Paradigm Shift in Manual Therapy? Evidence for a Central Nervous System Component in the Response to Passive Cervical Joint Mobilisation. Manual Therapy, 13, 387396. http://dx.doi.org/10.1016/j.math.2007.12.007

[22] Ragonese, J. (2009) A Randomised Trial Comparing Manual Physical Therapy to Therapeutic Exercises, to a Combination of Therapies, for the Treatment of Cervical Radiculopathy. Orthopaedic Physical Therapy Practice, 21, 71-76.

[23] Saranga, J., Green, A., Lewis, J. and Worsfold, C. (2003) Effect of a Cervical Lateral Glide on the Upper Limb Neurodynamic Test 1. Physiotherapy, 89, 678-684. http://dx.doi.org/10.1016/S0031-9406(05)60101-0

[24] Vicenzino, B., Collins, D. and Wright, A. (1994) Sudomotor Changes Induced by Neural Mobilisation Techniques in Asymptomatic Subjects. Journal of Manual and Manipulative Therapy, 2, 66-74. http://dx.doi.org/10.1179/jmt.1994.2.2.66

[25] Bogduk, N. and Mercer, S. (2000) Biomechanics of the Cervical Spine Part I: Normal Kinematics. Clinical Biomechanics, 15, 633-648. http://dx.doi.org/10.1016/S0268-0033(00)00034-6

[26] Wu, S. (2010) Segmental Percentage Contributions of Cervical Spine during Different Motion Ranges of Flexion and Extension. Journal of Spinal Disorders and Techniques, 23, 278-284. http://dx.doi.org/10.1097/BSD.0b013e3181a98d26

[27] Aquino, R., Caires, P., Furtado, F., et al. (2009) Applying Joint Mobilization at Different Cervical Vertebral Levels Does Not Influence Immediate Pain Reduction in Patients with Chronic Neck Pain: A Randomized Clinical Trial. Journal of Manual \& Manipulative Therapy, 17, 95-100. http://dx.doi.org/10.1179/106698109790824686

[28] Cleland, J., Childs, M., Mcrae, M., Palmer, J.A. and Stowell, T. (2005) Immediate Effects of Thoracic Manipulation in Patients with Neck Pain: A Randomized Clinical Trial. Manual Therapy, 10, 127-135. http://dx.doi.org/10.1016/j.math.2004.08.005

[29] Kanlyanaphotporn, R., Chiradejnant, A. and Vachalathiti, R. (2010) Immediate Effects of the Central Posteroanterior Mobilization Technique on Pain and Range of Motion in Patients with Mechanical Neck Pain. Disability and Rehabilitation, 32, 622-628. http://dx.doi.org/10.3109/09638280903204716

[30] Klaber-Moffett, J., Jackson, D., Richmond, S., et al. (2005) Randomised Trial of a Brief Physiotherapy Intervention Compared with Usual Physiotherapy for Neck Pain Patients: Outcomes and Patients' Preference. BMJ, 330, 75.

[31] Skargren, E.I., Carlsson, P.G. and Öberg, B.E. (1998) One-Year Follow-Up Comparison of the Cost-Effectiveness of Chiropractic and Physiotherapy as Primary Management for Back Pain: Sub-Group Analysis, Reoccurrence and Additional Health Care Utilization. Spine, 23, 1875-1883. http://dx.doi.org/10.1097/00007632-199809010-00016

[32] World Health Organisation (2002) Towards a Common Language for Function, Disability \& Health. ICF. http://www.int/classifications/icf/en

[33] Dworkin, R.H., Turk, D.C., Farrar, J.T., et al. (2005) Core Outcome Measures for Chronic Pain Clinical Trials: IMMPACT Recommendations. Pain, 113, 9-19. http://dx.doi.org/10.1016/j.pain.2004.09.012

[34] Mitchener, L.A., Snyder Valier, A.R. and McClure, P.W. (2013) Defining Substantial Clinical Benefit for PatientReported Outcome Tools for Shoulder Impingement Syndrome. Archives of Physical Medicine \& Rehabilitation, 94, 725-730. http://dx.doi.org/10.1016/j.apmr.2012.11.011

[35] Guyatt, G.H., King, D., Feeny, D.H., Stubbing, D. and Goldstein, R.S. (1999) Generic and Specific Measurement of Health Related Quality of Life in a Clinical Trial of Respiratory Rehabilitation. Journal of Clinical Epidemiology, 52, 187-192. http://dx.doi.org/10.1016/S0895-4356(98)00157-7

[36] Stock, S., Loisel, P. and Durand, M. (2003) IDVQ: L’indice d'impact de la douleur au cou et aux membres supérieurs sur la vie quotidienne (NULI: Neck and Upper Limb Index). Études et Recherches.

[37] Jenkinson, C., Stewart-Brown, S., Petersen, S. and Paice, C. (1999) Assessment of the SF-36 Version 2 in the United Kingdom. Journal of Epidemiology in Community Health, 53, 46-50. http://dx.doi.org/10.1136/jech.53.1.46

[38] Ware, R. (2002) User’s Manual for SF-36v2 Health Survey. 2nd Edition, Quality Metric Inc., Lincoln, 81-84.

[39] Phillips, C., Main, C. and Buck, R. (2008) Prioritising Pain in Policy Making: The Need for a Whole Systems Perspec- 
tive. Health Policy, 88, 166-175. http://dx.doi.org/10.1016/j.healthpol.2008.03.008

[40] US Department of Health and Human Services (2006) Common Terminology Criteria for Adverse Events. http://www.ctep.cancer.gov/protocolDevelopment/electronic_applications/docs/ctcaev3.pdf

[41] Berger, V.W. (2010) Testing for Baseline Balance: Can We Finally Get It Right? Journal of Clinical Epidemiology, 63, 939-940. http://dx.doi.org/10.1016/j.jclinepi.2010.02.014

[42] Fayers, P.M. and King, M.T. (2009) In Reply to Berger "Don’t Test for Baseline Imbalances unless They Are Known to Be Present?”. Quality of Life Research, 18, 401-402. http://dx.doi.org/10.1007/s11136-009-9458-2

[43] Allison, G.T., Nagy, B.M. and Hall, T. (2002) A Randomised Clinical Trial of Manual Therapy for Cervico-Brachial Pain Syndrome. Manual Therapy, 7, 95-102. http://dx.doi.org/10.1054/math.2002.0453

[44] Vicenzino, B., Collins, D. and Wright, A. (1996) The Initial Effects of a Cervical Spine Manipulative Therapy Treatment on the Pain and Dysfunction of Lateral Epicondyalgia. Pain, 68, 69-74. http://dx.doi.org/10.1016/S0304-3959(96)03221-6

[45] Vicenzino, B., Collins, D., Benson, H. and Wright, A. (1998) An Investigation of the Interrelationship between Manipulative Therapy-Induced Hypoalgesia and Sympathoexcitation. Journal of Manipulative and Physiological Therapeutics, 21, 448-453.

\section{Submit or recommend next manuscript to SCIRP and we will provide best service for you:}

Accepting pre-submission inquiries through Email, Facebook, LinkedIn, Twitter, etc. A wide selection of journals (inclusive of 9 subjects, more than 200 journals)

Providing 24-hour high-quality service

User-friendly online submission system

Fair and swift peer-review system

Efficient typesetting and proofreading procedure

Display of the result of downloads and visits, as well as the number of cited articles

Maximum dissemination of your research work

Submit your manuscript at: http://papersubmission.scirp.org/ 